Original Research Paper

\title{
Impact of Proven Viral Load on Common Cold Patients Treated with Pelargonium sidoides Preparation EPs 7630
}

\author{
${ }^{1}$ Tilman Keck, ${ }^{2}$ Andreas Strobl, ${ }^{3}$ Andreas Weinhaeusel and ${ }^{4}$ Berenike Stracke \\ ${ }^{I}$ Department of ENT Medicine, Head and Neck Surgery, Krankenhaus der Elisabethinen, Graz, Austria \\ ${ }^{2}$ Department of ENT Medicine, Head and Neck Surgery, Ordensklinikum Linz - Krankenhaus Barmherzige Schwestern, Linz, Austria \\ ${ }^{3}$ Health \& Environment Department, Molecular Diagnostics, AIT Austrian Institute of Technology GmbH, Wien, Austria \\ ${ }^{4}$ Clinical Research Department, Dr. Willmar Schwabe GmbH and Co. KG, Karlsruhe, Germany
}

Article history

Received: 16-08-2018

Revised: $17-08-2018$

Accepted: 07-11-2018

Corresponding Author:

Tilman Keck

Department of ENT Medicine,

Head and Neck Surgery,

Krankenhaus der Elisabethinen,

Graz, Austria

Tell +4331670631140

Fax +4331670631620

Email: Tilman.Keck@elisabethinen.at

\begin{abstract}
The Common Cold (CC) is an acute respiratory tract disorder with viral etiology in at least $90 \%$ of the cases. Nevertheless, molecular diagnostic testing often confirms the presence of viral Nucleic Acids (NA) in only about half of the patients. Based on the results of a non-comparative, interventional multicenter study we compare the course of CC between patients with and without proof of viral NA, who were treated with Pelargonium sidoides extract preparation EPs 7630. 120 adults with CC and at least 2 out of 10 common cold symptoms received $3 \times 1$ film-coated $20 \mathrm{mg}$ tablet EPs 7630 daily for 10 days. At baseline, tests for viral NA were performed. CC-associated symptoms and treatment satisfaction were evaluated after 5 days and at treatment end. Out of 119 patients with molecular nucleic acid based assessments, $61(61.3 \%)$ were tested positive for viral NA. Patients with and without proof of viral NA showed a similar time course of recovery from $\mathrm{CC}$ associated symptoms and had a comparable global outcome. In both subsets, less than $20 \%$ of the patients received concomitant paracetamol for antipyresis and only 2 required antibiotic treatment. Independently of whether or not viral NA was confirmed at baseline the course of CC treated with EPs 7630 was comparable. The results do not support the necessity to measure the viral load in order to treat CC patients effectively.
\end{abstract}

Keywords: Pelargonium Sidoides, Acute Rhinopharyngitis, Adults, Clinical Trial, Viral Analysis

\section{Introduction}

The Common Cold (CC) is a very common, acute respiratory tract infection. It has been estimated that at least $90 \%$ of all cases of CC are caused by viruses such as rhinovirus, corona virus, and respiratory syncytial virus (Dreschers et al., 2007; Herendeen and Szilagy, 2000; Niroumand and Grossman, 1998; van Kempen et al., 1999). The characteristic symptoms of CC include nasal congestion and discharge, sneezing, sore throat and cough. Complaints usually peak 2-3 days after the onset of infection, although some symptoms, e. g., cough and general discomfort, may persist for several weeks (Simasek and Blandino, 2007). The symptoms of CC are understood to be mainly attributable to the inflammatory host response to replicating viruses (Hasday et al., 2000). Viral infection of the nasal mucosa results in vasodilatation and increased vascular permeability, which in turn cause nasal obstruction and rhinorrhea. Cholinergic stimulation leads to increased mucous gland secretion and sneezing (Heikkinen and Järvinen, 2003).

Although CC is a predominantly trivial, self-limiting disorder, it may cause profound suffering and it is also associated with an important economic burden due to loss of productivity (Bertino, 2002; Birnbaum et al., 2002). Moreover, the inflammation of the nasal mucosa may cause an obstruction of the ostia of the paranasal sinuses and the Eustachian tubes, abetting the 
development of secondary inflammation such as sinusitis or otitis media (Pitrez and Pitrez, 2003). Pharmacological treatment of CC therefore appears to be justified. Since no antivirals have been licensed for the treatment of $\mathrm{CC}$ to date, current strategies aim at relieving symptoms, shortening the illness and reducing the risk of complications as well as the infectivity to other individuals (Arroll, 2011).

EPs $7630\left(\mathrm{EPs}^{\circledR} 7630\right.$ is the active ingredient of the product Umckaloabo ${ }^{\circledR}$ (ISO Arzneimittel, Ettlingen, Germany)) is a herbal drug preparation from the roots of the medicinal plant Pelargonium sidoides. Pharmacological activities of EPs 7630 and several of its isolated constituents have been demonstrated in invitro studies. They include a moderate direct antibacterial and antiviral action as well as notable immune-modulatory capabilities (Kolodziej, 2011; Moyo and Van Staden, 2014).

EPs 7630 has a prominent cytoprotective effect (Thäle et al., 2011) and has been shown to interfere with the replication of seasonal influenza A virus strains (H1N1, H3N2), respiratory syncytial virus, human coronavirus, parainfluenza virus and coxsackie virus (Michaelis et al., 2011). The non-specific, immunemodulatory effects of EPs 7630 appear to be mediated mainly by the release of tumor necrosis factor $\alpha$ and nitric oxides, the stimulation of interferon- $\beta$ and an increase in natural killer cell activity (Kayser et al., 2001; Kolodziej et al., 2003; Kolodziej and Kiderlen, 2007). Moreover, a recently completed in-vitro study showed that EPs 7630 strongly and dose-dependently induced the production of the pro-inflammatory cytokines TNF- $\alpha$ and IL- 6 in human blood immune cells, suggesting that the extract acts as an immunostimulant (Witte et al., 2015).

Clinically, EPs 7630 has been demonstrated to be efficacious in the symptomatic treatment of acute respiratory tract infections (Agbabiaka et al., 2008; Matthys et al., 2014; Timmer et al., 2013). While the majority of controlled clinical trials were performed in acute bronchitis, studies are also available for acute rhinosinusitis, acute tonsillopharyngitis and CC. Moreover, therapeutic evidence for adults having $\mathrm{CC}$ with acute rhinosinusitis as an overlapping symptom has also been included in a European guideline, including a recommendation for viral and post-viral acute rhinosinusitis directly based on category I evidence (Fokkens et al., 2012).

In a recently completed clinical trial the tolerability and course of EPs 7630 treatment in adult patients suffering from CC were observed (Keck et al., 2015). The study procedures involved virus testing at baseline. We investigate the association between the detection of virus NA at the start of treatment and the subsequent course of the disease specific symptoms.

\section{Materials and Methods}

\section{Design and Participants}

We report on an open-label, non-comparative, interventional multicenter study in patients suffering from CC, who were treated with EPs 7630 for 10 days. Assessments were performed at baseline, after 5 days, as well as at end of treatment. Participants maintained a diary to record the intensity of their disease-related symptoms on a daily basis.

The protocol was reviewed and approved by an independent ethics committee. All patients provided written informed consent. The principles of Good Clinical Practice and the Declaration of Helsinki were adhered to.

Eligible participants had to be female or male outpatients $\geq 18$ years of age, with a clinical diagnosis of CC and at least 2 out of the following 10 pre-defined symptoms (Common Cold Symptoms, CCS): nasal discharge, sore throat, nasal congestion, sneezing, scratchy throat, hoarseness, coughing, headache, malaise, fever. Patients with obstructive anatomic nasopharyngeal lesions (e.g., nasal polyps), severe septal deviations, previous or planned surgery of the nose or paranasal sinuses, chronic pulmonary diseases, allergic rhinitis, conditions known to cause sore throat (e.g., tonsillopharyngitis, drugs, aphthous ulcers, candida), or any acute respiratory tract disease other than $\mathrm{CC}$, were excluded from participation.

\section{Treatments}

The study medication was EPs 7630, a herbal drug preparation from the roots of Pelargonium sidoides (1: 8$10)$, extraction solvent: ethanol $11 \%(\mathrm{w} / \mathrm{w})$. Patients had to administer $3 \times 1$ film-coated tablets per day (Marketed product used in this trial: Kaloba ${ }^{\circledR}$ (Austroplant Arzneimittel, Vienna, Austria).), each containing $20 \mathrm{mg}$ of dried EPs 7630, for 10 consecutive days.

Treatment compliance was assessed by counting the number of remaining film-coated tablets at study exit. Except for paracetamol taken in case of fever $>38.5^{\circ} \mathrm{C}$, up to a maximum dose of $500 \mathrm{mg}$ every 6 hours, concomitant $\mathrm{CC}$ medications that might impair the interpretation of trial results was not allowed.

\section{Molecular Diagnostic Assays}

Molecular testing for viruses was performed at baseline, by taking a nasopharyngeal swab from both nostrils with a sterile brush. Total nucleic acid (DNA and RNA) was isolated from the samples using the QIAamp nucleic acid isolation kit (Qiagen, Hilden, Germany). The amount of isolated nucleic acid was measured by absorbance. For viral testing, an aliquot was applied to the $\mathrm{XTAG}^{\circledR}$ Respiratory Viral Panel (RVP) FAST assay 
(Abbott, Vienna, Austria). cDNA were generated from RNA by Reverse Transcription (RT) and then amplified by PCR. Subsequent to RT-PCR the amplicons were hybridized onto virus species specific DNA probes immobilized on Luminex beads. Virus species were identified using Luminex technology, with signal measurements of virus specific hybridization signals specified for the $\mathrm{xTAG}^{\circledR}$ RVP FAST assay. Viruses detectable by the assay include influenza $\mathrm{A}$ and $\mathrm{B}$, Respiratory Syncytial Virus (RSV), corona virus, parainfluenza virus, human metapneumovirus, enterorhinovirus, adenovirus and human bocavirus. Testing of bacterial DNA was done using PCR amplification targeting a 738bp 16S rRNA gene sequence and microarray hybridization using the AIT Chip HD PathoID test enabling detection of 73 known human bacterial pathogens (Austrian Institute of Technology).

\section{Measures for Course of Disease}

Patient information included demographic, anthropometric and medical history data. The course of $\mathrm{CC}$ was assessed at each visit based on investigator ratings of each of a set of $10 \mathrm{CCS}$ on a 4-point verbal rating scale ranging from 0 ('not present') to 3 ('severe'), which are summed up to a total score based on a scale developed by Jackson and colleagues (Jackson et al., 1958). Observer assessments also included 8 additional CC-relevant complaints (CRC; pulmonary rales at auscultation, sputum production, chest pain during coughing, chilliness, exhaustion, loss of appetite, diarrhea and muscle aches) which the investigators rated using the same scale and which were summed up to a separate total score. Moreover, an over-all symptom score was computed from the $10 \mathrm{CCS}$ and the $8 \mathrm{CRC}$.

Recovery from $\mathrm{CC}$ (rated by patients and investigators) as well as the patients' satisfaction with the treatment course were assessed using the Integrative Medicine Outcomes Scale (IMOS) and the Integrative Medicine Patient Satisfaction Scale (IMPSS), respectively (Steinsbekk et al., 1999). Moreover, the patient diary included daily global assessments of how ill a patient felt. Further measures to assess the course of the disease were the number of days off work or education, the need for antibiotic treatment and the use of paracetamol.

\section{Statistical Methods, Sample Size}

We performed descriptive post-hoc comparisons between patients with viral NA proof at baseline and those in whom no viral NA could be confirmed. Within each subset, descriptive summary statistics were computed and $\mathrm{p}$-values and 95\% Confidence Intervals (CIs) were determined for change over time using. For eligibility of the analyses of course of $\mathrm{CC}$, at least one post-baseline assessment of the analyzed outcome was required (Full Analysis Set, FAS). Missing data were imputed by carrying forward the last valid observation provided that at least one post-baseline assessment had been performed.

For statistical tests two-sided p-values up to 0.05 were considered to be descriptively significant. A total of 120 patients were planned to be treated based on practical considerations.

\section{Results}

\section{Participant Characteristics}

A total of 120 participants were included and treated in the out-patient clinics of 8 hospitals in Austria between January 2011 and November 2012. One hundred and seventeen participants completed the trial as scheduled. All patients included were analyzed for parameters to assess the course of the disease in the FAS.

For 119 patients, swab samples were available for analysis. Virus NA was detected in the samples of 61 patients $(51.3 \%$ of 119 ; Fig. 1). The most frequently detected viruses were enterovirus/rhinovirus $(29 \%$ of the samples), followed by corona HKU1 virus (11\%) and by Corona OC43 virus (7\%). The samples of 50 of the 61 patients $(82.0 \%)$ showed infection with 1 virus species whereas more than 1 species was found in the samples of the remaining 11 patients $(18 \%)$.

The following sections present the results of the 119 patients for whom nasopharyngeal swab samples were available for analysis. The results of the pre-planned safety and disease course analyses performed on all treated patients $(n=120)$ have been reported elsewhere (Keck et al., 2015).

\section{Baseline Characteristics}

Table 1 shows the study participants' demographic and anthropometric characteristics. On average, patients with proof of viral NA were about 5 years younger than those without confirmed viral NA, but only minor subgroup differences were observed otherwise. Although ethnic group was not a selection criterion, all subjects except 1 were Caucasians. About $75 \%$ of the subjects in both subsets reported to drink alcohol occasionally or regularly. $55.7 \%$ of the patients with proof of viral NA and $41.4 \%$ of those without confirmed viral NA were current smokers or ex-smokers.

At baseline, patients with viral NA had been suffering from symptoms of Acute Rhinopharyngitis (ARP) for an average of $50.1 \pm 16.4$ hours (mean $\pm \mathrm{SD}$; range $16-72$ hours) compared to $42.3 \pm 19.6$ hours (range: 6-72 hours) in patients without confirmed viral NA. The baseline severity of CCS and CRC showed no monotonic association with symptom duration upon enrolment. 
Table 1: Baseline characteristics (number (\%) of patients or mean \pm SD, by pathogen detection)

\begin{tabular}{lll}
\hline Virus NA & Confirmed $(\mathrm{n}=61)$ & Not confirmed $(\mathrm{n}=58)$ \\
\hline Female & $44(72.1 \%)$ & $39(67.2 \%)$ \\
Male & $17(27.9 \%)$ & $19(32.8 \%)$ \\
Age (years) & $36.3 \pm 12.4$ & $41.4 \pm 14.5$ \\
Height $(\mathrm{cm})$ & $171.9 \pm 8.7$ & $170.7 \pm 7.5$ \\
Weight (kg) & $71.7 \pm 13.4$ & $74.7 \pm 15.4$ \\
Duration of ARP symptoms [hours] & $50.1 \pm 16.4$ & $42.3 \pm 19.6$ \\
\hline
\end{tabular}

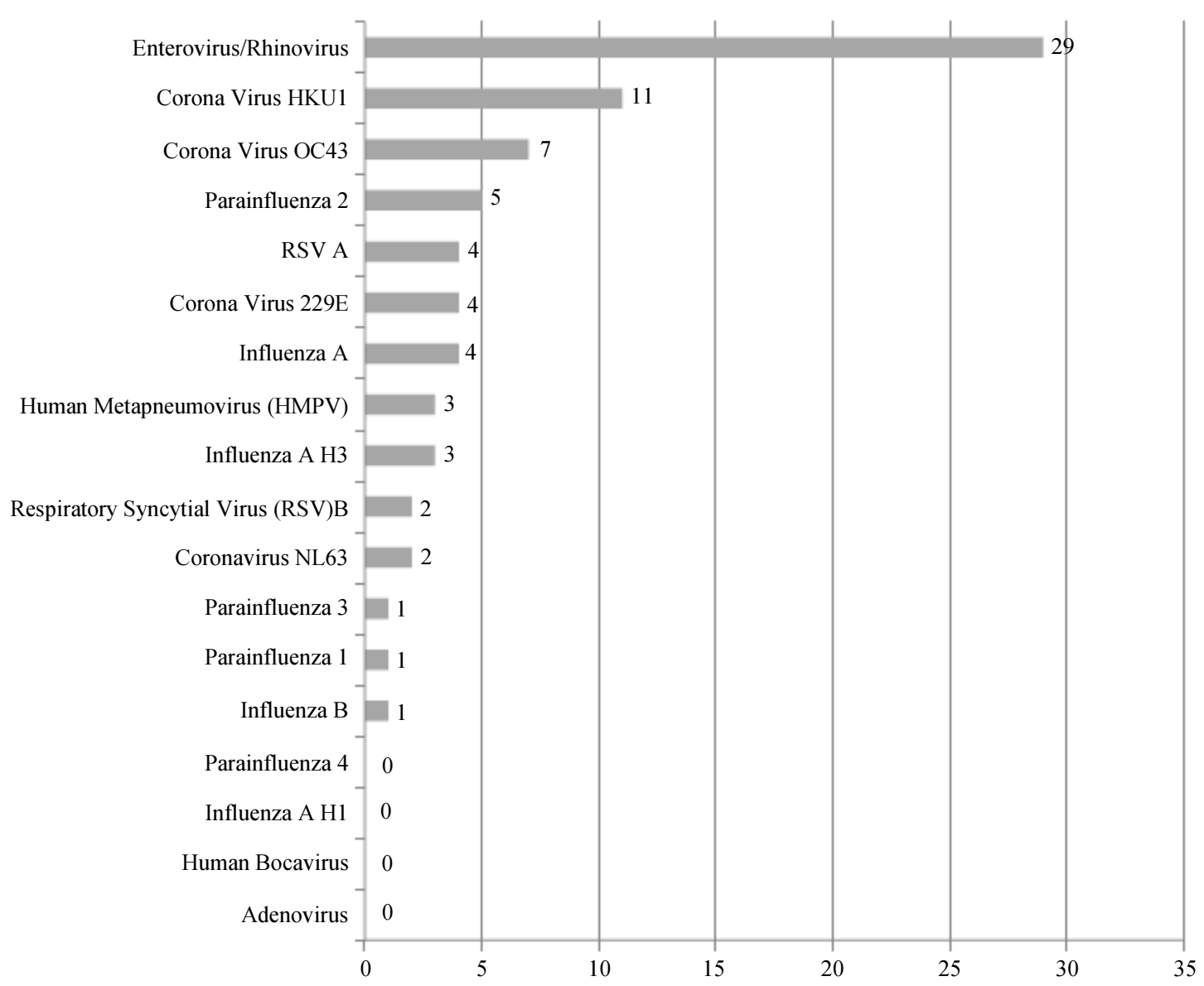

Fig. 1: Detection of virus NA in the nasal swab samples of 119 patients ( $\%$ of patients, by virus species)

\section{Time Course of Common Cold Associated Symptoms}

According to the investigators' rating, patients with proof of viral NA showed a slightly higher CCS total score at baseline than those without confirmed viral NA, but a comparable time course of symptom recovery was observed for both subsets. Differences between the subsets regarding the CRC total score were also marginal throughout the period of observation (Fig. 2). For both CCS and CRC, only a minimum symptom burden remained at treatment end, with individual maximum scores of 2 and 1 points for CCS (theoretical maximum score of scale: 30 points) and of 2 and 2 points for CRC (theoretical maximum: 24 points), for patients with or without confirmed viral NA, respectively.

Table 2 presents the average intraindividual change of the CCS and CRC total scores. According to the investigators' rating, patients with and without proof of virus NA achieved descriptively significant average score reductions by about $50 \%$ of the baseline value already after 5 days of treatment and continued to improve until treatment end. Since the majority of patients in both subsets had only minimal, if any residual symptoms at day 10 , the observed differences in average change between patients with and without proof of virus NA, particularly for CCS, likely reflect baseline differences rather than differences in the time course of symptom recovery. 
Tilman Keck et al. / American Journal of Infectious Diseases 2018, 14 (3): 93.102 DOI: 10.3844/ajidsp.2018.93.102

Table 2: Common cold associated symptoms - baseline total score and change between baseline and subsequent visits (investigator ratings; mean \pm SD and Wilcoxon test for change versus baseline; FAS)

\begin{tabular}{|c|c|c|c|c|c|c|c|}
\hline \multirow[t]{2}{*}{ Virus NA } & & \multicolumn{3}{|c|}{ Confirmed } & \multicolumn{3}{|c|}{ Not confirmed } \\
\hline & & $\mathrm{n}$ & Mean \pm SD & $\mathrm{p}$ & $\mathrm{n}$ & Mean \pm SD & $\mathrm{p}$ \\
\hline Common Cold & Baseline & 61 & $11.5 \pm 3.4$ & & 58 & $10.1 \pm 3.7$ & \\
\hline \multirow[t]{2}{*}{ Symptoms (CCS) } & Change, day 5 & 59 & $-6.2 \pm 4.4$ & $<0.01$ & 57 & $-5.0 \pm 2.9$ & $<0.01$ \\
\hline & Change, day 10 & 60 & $-9.5 \pm 3.8$ & $<0.01$ & 55 & $-7.9 \pm 3.9$ & $<0.01$ \\
\hline Further common & Baseline & 61 & $3.3 \pm 2.4$ & 58 & $3.5 \pm 3.0$ & & \\
\hline Cold Relevant & Change, day 5 & 59 & $-2.0 \pm 2.0$ & $<0.01$ & 57 & $-2.2 \pm 2.8$ & $<0.01$ \\
\hline Complaints (CRC) & Change, day 10 & 60 & $-2.6 \pm 2.1$ & $<0.01$ & 55 & $-3.0 \pm 3.1$ & $<0.01$ \\
\hline Grand total & Baseline & 61 & $14.8 \pm 4.9$ & 58 & $13.6 \pm 5.8$ & & \\
\hline \multirow[t]{2}{*}{$(\mathrm{CCS}+\mathrm{CRC})$} & Change, day 5 & 59 & $-8.2 \pm 5.3$ & $<0.01$ & 57 & $-7.2 \pm 5.0$ & $<0.01$ \\
\hline & Change, day 10 & 60 & $-12.0 \pm 5.0$ & $<0.01$ & 55 & $-10.9 \pm 6.1$ & $<0.01$ \\
\hline
\end{tabular}
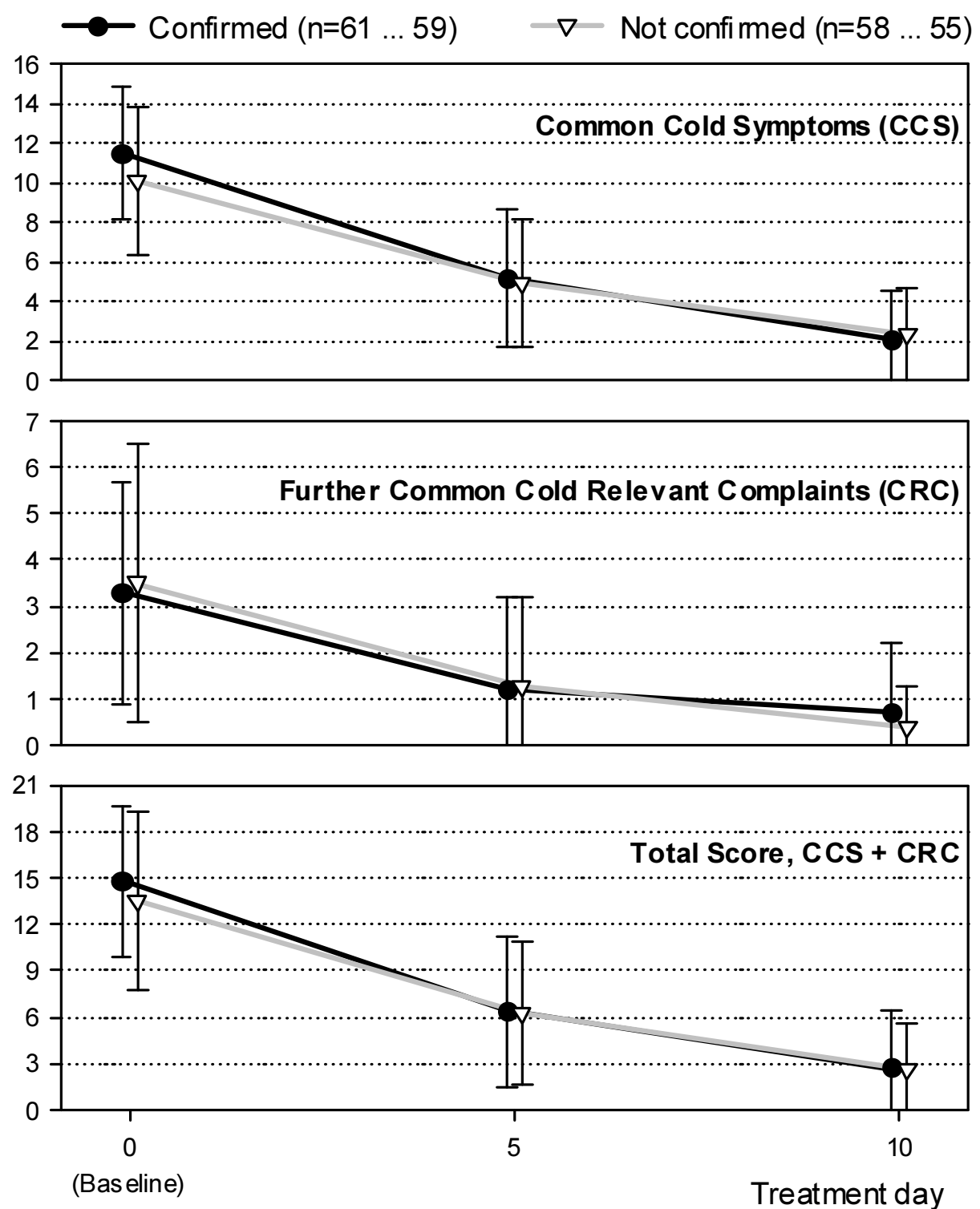

Fig. 2: Time course of common cold symptoms and further common cold relevant complaints, by confirmation of virus NA (investigator ratings; mean $\pm \mathrm{SD}$; FAS) 
The results for the observer ratings of CCS were supported by the patients' self-ratings obtained in the diary on a daily basis (data not shown).

\section{Global Disease Course Measurements, Patients' Impression of Treatment Satisfaction}

According to the investigators' IMOS ratings, about half of the patients with confirmed viral NA and close to $40 \%$ of patients without proof of viral NA showed at least major improvements after 5 days of treatment with EPs 7630 (Fig. 3). At treatment end, 28 of 60 patients with valid data and proof of viral NA (46.7\%) were completely recovered and $22(36.7 \%)$ showed major improvement, compared to $21(37.5 \%)$ and $26(46.4 \%)$ out of 56 patients in the subset without confirmed viral NA. The data suggest that although the total rates of patients with major improvements or complete recovery were comparable for patients with or without confirmed viral NA, those with confirmed viral infection had a higher complete recovery rate at treatment end. The investigators' assessments were supported by the patients' IMOS self-ratings which also indicated a slightly higher rate of patients with a favorable impression of the disease course after 5 and 10 days. These results were in line with the daily self-assessments if severity of illness obtained in the patient diaries: by day 5 (viral NA) and day 4 (no confirmed viral NA), more than $50 \%$ of the patients with valid data reported that they felt only very mildly ill or not ill at all. The rates increased to at or above $90 \%$ by day 8 (viral NA) and 10 (no proof of viral NA), respectively.

The results of the IMOS assessments were also consistent with the treatment satisfaction self-ratings obtained with the IMPSS (Fig. 3): 22 of 60 patients with valid data and proof of viral NA (36.1\%) were very much satisfied and $18(29.5 \%)$ were satisfied, compared to $15(25.9 \%)$ and $19(32.8 \%)$ out of 58 patients in the subset without proof of viral NA. In each subset, 1 patient indicated that she/he was dissatisfied or very dissatisfied with the disease outcome at the end of the observational period.

\section{Need for Antibiotics or Paracetamol}

Paracetamol, which was allowed in accordance with the study protocol in case of fever $>38.5^{\circ} \mathrm{C}$, was taken at least once by 11 of the 61 patients $(18.0 \%)$ with proof of viral NA and by 11 of the 58 patients $(19.0 \%)$ without confirmed viral NA. One patient in each subset $(1.6 \%$ and $1.7 \%$ for patients with and without proof of viral NA, respectively) received antibiotic treatment in accordance with the investigator's medical judgment.

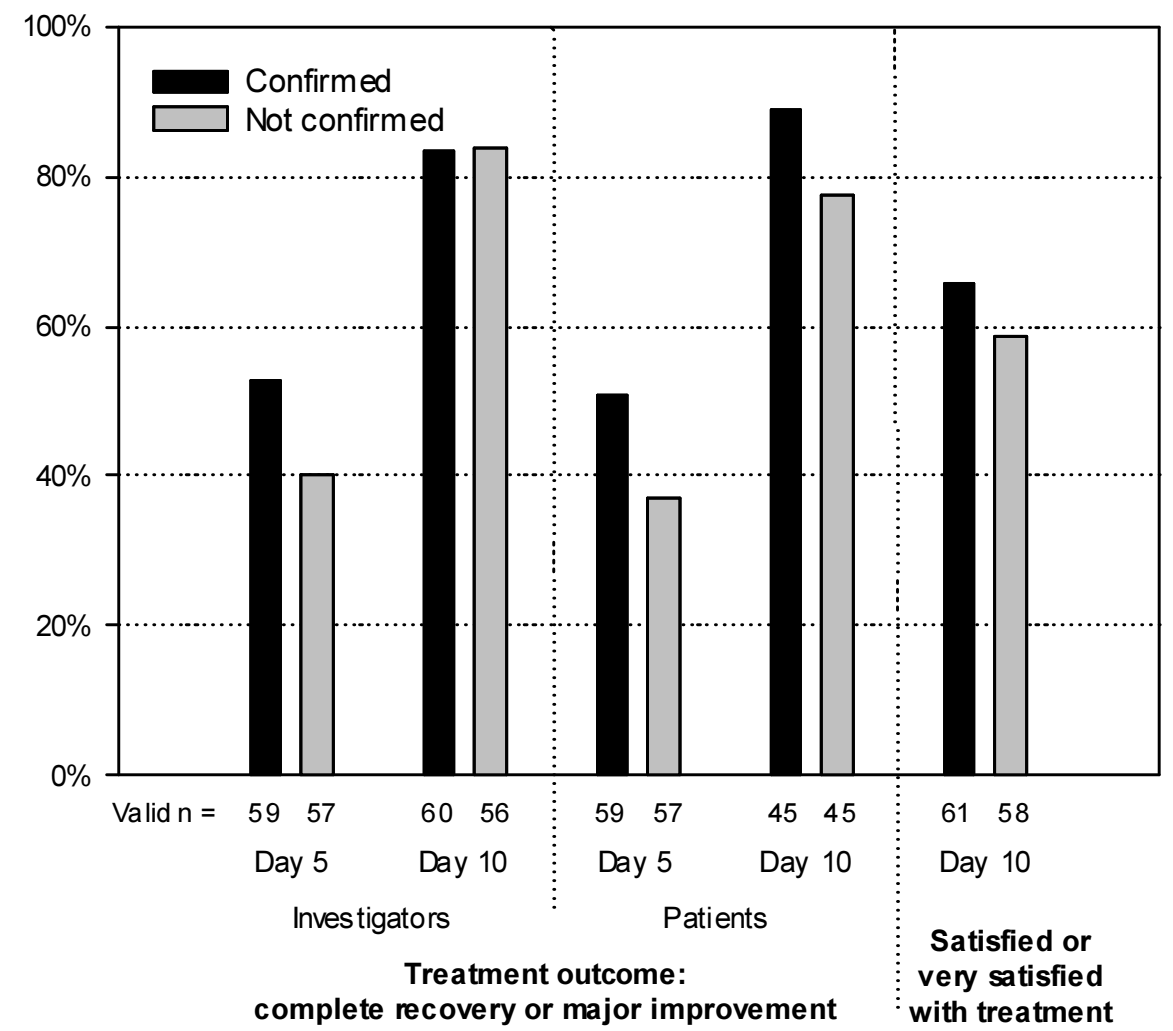

Fig. 3: Treatment outcome (IMOS) and satisfaction with treatment - \% of patients with a favorable response, by confirmation of virus NA (FAS) 


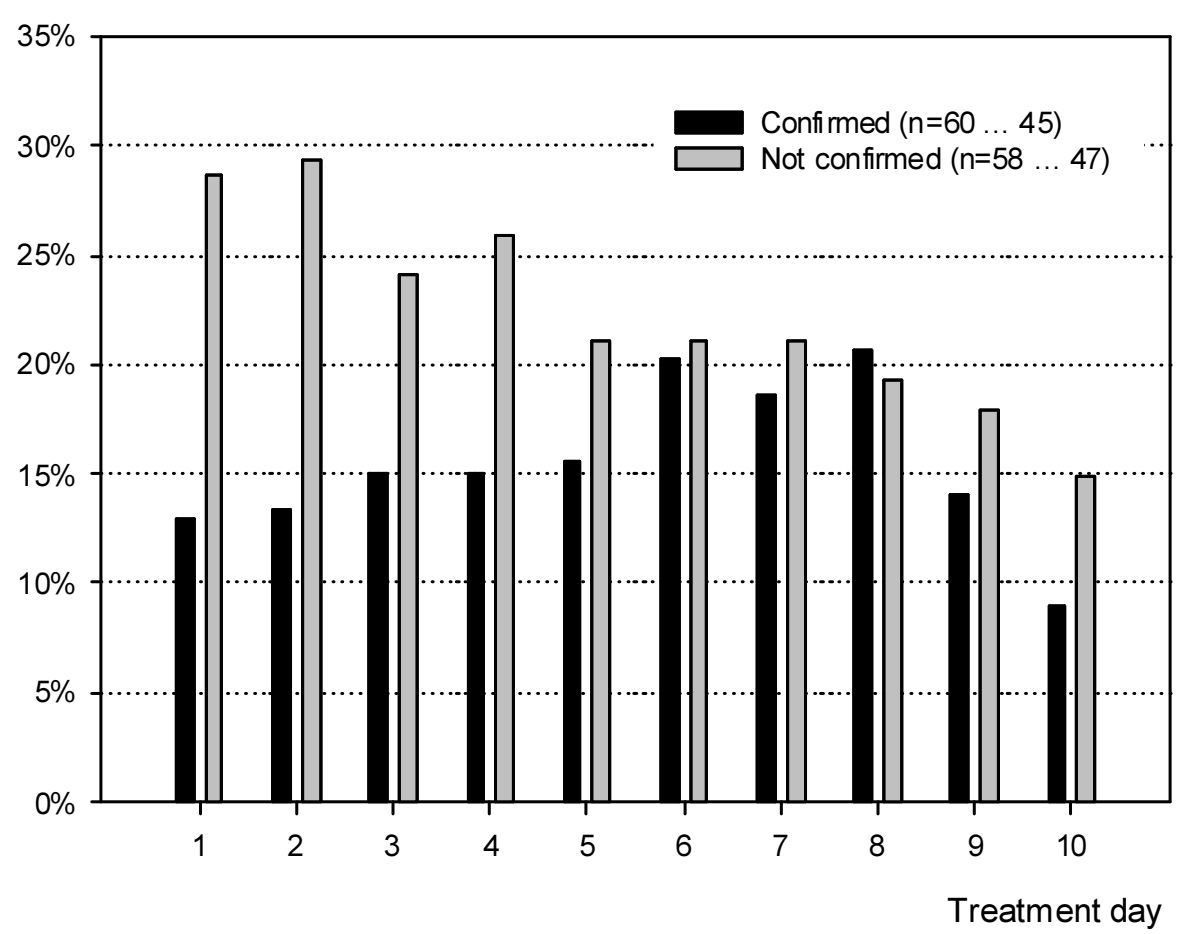

Fig. 4: Percent of patients who did not attend work or school/college, by confirmation of virus NA (FAS)

\section{Inability to Work}

In the subset with proof of viral NA between $13 \%$ and $21 \%$ of the patients did not attend work or school/college throughout the majority of days of observation. The rate decreased to $8.9 \%$ by day 10 . Patients without confirmed viral NA had initially higher non-attendance rates just below $30 \%$ which decreased gradually to $14.9 \%$ at the end of the observational period (Fig. 4).

The average intraindividual number of days off work or school/college was $1.2 \pm 2.7$ days in patients with proof of viral NA and $2.3 \pm 3.8$ days in patients without confirmed viral NA, with maximum values of 10 and 11 days, respectively.

\section{Discussion}

Acute respiratory tract infections such as $\mathrm{CC}$ are almost exclusively viral in origin (Dreschers et al., 2007; Herendeen and Szilagy, 2000; Mäkelä et al., 1998; Niroumand and Grossman, 1998; van Kempen et al., 1999). Despite the fact that highly sensitive assays have become available during recent years (Ginocchio and McAdam, 2011), diagnostic tests still vary widely with regard to their sensitivity and specificity. The virus NA detection rate has been shown to decrease significantly with increasing symptom duration (Brittain-Long et al., 2010). In many investigations, only about half of the infected patients show a positive virus test result (e.g., Brittain-Long et al., 2010; Lam et al., 2007), although detection rates exceeding $90 \%$ have also been reported when patients were assessed within 48 hours after the onset of the first symptoms. Moreover, virus detection rates have also been found to vary with the type of specimen (e.g., nasopharyngeal vs. throat/saliva) (Robinson et al., 2008) as well as with the type of transport medium used (Walsh et al., 2008). These observations are consistent with the analysis of the swab obtained at baseline of our trial, which confirmed viral NA in about half of the samples. It is important to note, however, that the results do by no means imply that patients in whom the test for viral NA remained negative were not suffering from viral infection.

As regards the clinical manifestations and course of CC in patients treated with EPs 7630 , our analyses found no evidence of major differences between patients in whom viral NA could or could not be confirmed at baseline. Both subsets showed a comparably favorable course, with significant improvement of symptoms already during the first 5 days while the patients had largely recovered after 10 days. The results also indicate that patients with and without proof of viral NA were most probably not suffering from different clinical conditions, but that those in whom no virus could be confirmed may either have been already in a post-viral state, or may have had a false negative test.

Paracetamol is among the most widely used medications in CC ( $\mathrm{Li}$ et al., 2013). In this trial, comedication with paracetamol was permitted for antipyresis, but was actually used by less than $20 \%$ of patients in both subsets. Since EPs 7630 has no known direct antipyretic effect, we interpret the comparatively 
low use of paracetamol as an indicator of a favorable course of the infection during the administration of the herbal medicinal product, so that only a minority of patients developed fever in a range that prompted antipyresis. Remarkably, only 1 patient in each subset was switched to an antibiotic.

Our investigation thus shows that patients with or without proof of virus NA treated with EPs 7630 exhibited a similarly favorable course of CC symptoms and rarely required additional or different drug treatment. In acute respiratory tract disorders such as $\mathrm{CC}$, testing for viral NA therefore appears to be dispensable in clinical routine practice because the results of the test will probably not modify the appropriate treatment to be administered.

A limitation of the study is that the design did not include a placebo control group and thus the treatment effect in a self-limiting condition like $\mathrm{CC}$ is difficult to assess. However, as argued previously (Keck et al., 2015), the extent of symptom relief observed in this study was comparable to that in a double-blind, randomized trial performed by Lizogub and colleagues (Lizogub et al., 2007) in CC, in which EPs 7630 was significantly superior to placebo.

\section{Conclusion}

Our analysis showed no association between the presence of virus NA confirmed by a microbiological assessment performed at baseline and the course of CC in patients treated with EPs 7630. Although the design of this study was not intended for demonstrating treatment efficacy, the favorable course of clinical symptoms is comparable to the course observed earlier in the active treatment group of a placebo-controlled trial (Keck et al., 2015). The results may also be explained by the antiviral effect of EPs 7630 that has been demonstrated in preclinical investigations (Kolodziej, 2011; Moyo and Van Staden, 2014).

\section{Acknowledgment}

Medical writing services were provided by Dr. Andreas Völp, Psy Consult Scientific Services, Frankfurt, Germany.

\section{Funding}

The study as well as medical writing services were supported by Dr. Willmar Schwabe GmbH \& Co. KG, Karlsruhe, Germany.

\section{Author Contributions}

Tilman Keck: Was principal investigator of the study and provided input into the study design, the interpretation of the data and the drafting of the manuscript.
Andreas Strobl: Was investigator of the study and provided input into the study design, the interpretation of the data and the drafting of the manuscript.

Andreas Weinhaeusel: Was responsible for the molecular diagnostics and was involved in the interpretation of the data and the drafting of the manuscript.

Berenike Stracke: Was involved in the interpretation of the data and helped to draft the manuscript.

\section{Conflict of Interest}

Tilman Keck, Andreas Strobl and Andreas Weinhaeusel have received honoraria from Dr. Willmar Schwabe GmbH and Co. KG, Karlsruhe, Germany. Berenike Stracke is an employee of Dr. Willmar Schwabe GmbH and Co. KG, Karlsruhe, Germany.

\section{References}

Agbabiaka, T.B., R. Guo and E. Ernst, 2008. Pelargonium sidoides for acute bronchitis: A systematic review and meta-analysis. Phytomedicine, 15: 378-385.

DOI: $10.1016 /$ j.phymed.2007.11.023

Arroll, B., 2011. Common cold. Clin. Evid., 03: 1510-1510. PMID:21406124

Bertino, J.S., 2002. Cost burden of viral respiratory infections: Issues for formulary decision makers. Am. J. Med., 112 Suppl 6A: 42S-49S. DOI: $10.1016 / \mathrm{S} 0002-9343(01) 01063-4$

Birnbaum, H.G., M. Morley, P.E. Greenberg and G.L. Colice, 2002. Economic burden of respiratory infections in an employed population. Chest, 122: 603-611. DOI: 10.1378/chest.122.2.603

Brittain-Long, R., J. Westin, S. Olofsson, M. Lindh and L.M. Andersson, 2010. Prospective evaluation of a novel multiplex real-time PCR assay for detection of fifteen respiratory pathogens-duration of symptoms significantly affects detection rate. J. Clin. Virol., 47: 263-267. DOI: 10.1016/j.jcv.2009.12.010

Dreschers, S., P. Franz, C.A. Dumitru, B. Wilker and K. Jahnke et al., 2007. Infections with human rhinovirus induce the formation of distinct functional membrane domains. Cell Physiol. Biochem., 20: 241-254. DOI: 10.1159/000104170

Fokkens, W.J., V.J. Lund, J. Mullol, C. Bachert and I. Alobid et al., 2012. European position paper on rhinosinusitis and nasal polyps 2012. Rhinology, 50: 3-3 p preceding table of contents, 1-298.

Ginocchio, C.C. and A.J. McAdam, 2011. Current best practices for respiratory virus testing. J. Clin. Microbiol., 49: S44-S48. DOI: 10.1128/JCM.00698-11

Hasday, J.D., K.D. Fairchild and C. Shanholtz, 2000. The role of fever in the infected host. Microbes Infect., 2: 1891-1904. DOI: $10.1016 / \mathrm{S} 1286-4579(00) 01337-\mathrm{X}$ 
Heikkinen, T. and A. Järvinen, 2003. The common cold. Lancet, 361: 51-59. DOI: $10.1016 / \mathrm{S} 0140-6736(03) 12162-9$

Herendeen, N.E. and P.G. Szilagy, 2000. Infections of the Upper Respiratory Tract. In: Nelson Textbook of Pediatrics, Behrman, R.E., R.M. Kliegman and H.B. Jenson (Eds.), W. B. Saunders, Philadelphia, pp: 1261-1266.

Jackson, G.G., H.F. Dowling, I.G. Spiesman and A.V. Boand, 1958. Transmission of the common cold to volunteers under controlled conditions. I. The common cold as a clinical entity. AMA Arch. Intern. Med., 101: 267-278.

DOI: $10.1001 /$ archinte. 1958.00260140099015

Kayser, O., H. Kolodziej and A.F. Kiderlen, 2001. Immunomodulatory principles of Pelargonium sidoides. Phytother. Res., 15: 122-126. PMID: 11268110

Keck, T., A. Strobl and B. Stracke, 2015. Tolerability and treatment effects of Pelargonium sidoides preparation EPs 7630 in adults suffering from acute rhinopharyngitis - a prospective, open-label trial, Altern. Integ. Med., pp: 204.

Kolodziej, H., 2011. Antimicrobial, antiviral and immunomodulatory activity studies of Pelargonium sidoides $\left(\mathrm{EPS}^{\circledR}\right.$ 7630) in the context of health promotion. Pharmaceuticals, 4: 1295-1314. DOI: $10.3390 / \mathrm{ph} 4101295$

Kolodziej, H., O. Kayser, O.A. Radtke, A.F. Kiderlen and E. Koch, 2003. Pharmacological profile of extracts of Pelargonium sidoides and their constituents. Phytomedicine, 10 Suppl 4: 18-24. PMID: 12807338

Kolodziej, H. and A.F. Kiderlen, 2007. In vitro evaluation of antibacterial and immunomodulatory activities of Pelargonium reniforme, Pelargonium sidoides and the related herbal drug preparation EPs ${ }^{\circledR}$ 7630. Phytomedicine, 14 Suppl 6: 18-26.

DOI: 10.1016/j.phymed.2006.11.020

Lam, W.Y., A.C. Yeung, J.W. Tang, M. Ip and E.W. Chan et al., 2007. Rapid multiplex nested PCR for detection of respiratory viruses. J. Clin. Microbiol., 45: 3631-3640.

DOI: $10.1128 / \mathrm{JCM} .00280-07$

Li, S., J. Yue, B.R. Dong, M. Yang and X. Lin et al., 2013. Acetaminophen (paracetamol) for the common cold in adults. Cochrane Database Syst. Rev., 7: CD008800.

DOI: $10.1002 / 14651858 . C D 008800 . p u b 2$

Lizogub, V.G., D.S. Riley and M. Heger, 2007. Efficacy of a Pelargonium sidoides preparation in patients with the common cold: A randomized, double blind, placebo-controlled clinical trial. Explore, 3: 573-584.

DOI: $10.1016 /$ j.explore.2007.09.004
Mäkelä, M.J., T. Puhakka, O. Ruuskanen, M. Leinonen and P. Saikku et al., 1998. Viruses and bacteria in the etiology of the common cold. J. Clin. Microbiol., 36: 539-542. PMID: 9466772

Matthys, H., F.A. Malek and W. Kamin, 2014. EPs ${ }^{\circledR}$ 7630 in acute respiratory tract infections - a systematic review and meta-analysis of randomised clinical trials. Forsch. Komplementmed., 21: 57-58. DOI: $10.1159 / 000362397$

Michaelis, M., H.W. Doerr and J. Cinatl, Jr., 2011. Investigation of the influence of $\operatorname{EPs}^{\circledR} 7630$, a herbal drug preparation from Pelargonium sidoides, on replication of a broad panel of respiratory viruses. Phytomedicine, 18: 384-386.

DOI: 10.1016/j.phymed.2010.09.008

Moyo, M. and J. Van Staden, 2014. Medicinal properties and conservation of Pelargonium sidoides DC. J. Ethnopharmacol., 152: 243-255. DOI: 10.1016/j.jep.2014.01.009

Niroumand, M. and R.F. Grossman, 1998. Airway infection. Infect. Dis. Clin. North Am., 12: 671-688. PMID: 9779384

Pitrez, P.M. and J.L. Pitrez, 2003. Acute upper respiratory tract infections: Outpatient diagnosis and treatment [in Portugese]. J. Pediatr. (Rio. J.), 79 Suppl 1: S77-S86. PMID: 14506520

Robinson, J.L., B.E. Lee, S. Kothapalli, W.R. Craig and J.D. Fox, 2008. Use of throat swab or saliva specimens for detection of respiratory viruses in children. Clin. Infect. Dis., 46: e61-64.

DOI: $10.1086 / 529386$

Simasek, M. and D.A. Blandino, 2007. Treatment of the common cold. Am. Fam. Physician, 75: 515-520. PMID: 17323712

Steinsbekk, A., J. Biolchini, M. Heger, C. Rezzani and N. Tsamis et al., 1999. Data collection in homeopathic practice. A proposal for an international standard. HomInt R\&D Newsletter, 2: 3-23.

Thäle, C., A.F. Kiderlen and H. Kolodziej, 2011. Anti-infective activities of Pelargonium sidoides $\left(\mathrm{EPs}^{\circledR} 7630\right)$ : Effects of induced NO production on Leishmania major in infected macrophages and antiviral effects as assessed in a fibroblast-virus protection assay. Planta Med., 77: 718-725. DOI: $10.1055 / \mathrm{s}-0030-1250567$

Timmer, A., J. Günther, E. Motschall, G. Rücker and G. Antes et al., 2013. Pelargonium sidoides extract for treating acute respiratory tract infections. Cochrane Database Syst. Rev., 10: CD006323.

DOI: 10.1002/14651858.CD006323.pub3

van Kempen, M., C. Bachert and P. Van Cauwenberge, 1999. An update on the pathophysiology of rhinovirus upper respiratory tract infections. Rhinology, 37: 97-103. PMID: 10567986 
Walsh, P., C.L. Overmyer, K. Pham, S. Michaelson and L. Gofman et al., 2008. Comparison of respiratory virus detection rates for infants and toddlers by use of flocked swabs, saline aspirates and saline aspirates mixed in universal transport medium for room temperature storage and shipping. J. Clin. Microbiol., 46: 2374-2376.

DOI: $10.1128 / J C M .00714-08$
Witte, K., E. Koch, H.D. Volk, K. Wolk and R. Sabat, 2015. The Pelargonium sidoides extract EPs 7630 drives the innate immune defense by activating selected MAP kinase pathways in human monocytes. PloS One, 10: e0138075.

DOI: 10.1371 /journal.pone. 0138075 